\title{
Effect of gargling with tea and ingredients of tea on the prevention of influenza infection: a meta-analysis
}

Kazuki Ide, Hiroshi Yamada* and Yohei Kawasaki

\begin{abstract}
Background: Influenza viruses can spread easily from person to person, and annual influenza epidemics are serious public health issues worldwide. Non-pharmaceutical public health interventions could potentially be effective for combatting influenza epidemics, but combined interventions and/or interventions with greater effectiveness are needed. Experimental studies have reported that tea and its ingredients (especially catechins) have antiviral activities. Although several clinical studies have investigated the use of tea or its ingredients to prevent influenza infections, the effect of gargling these substances has remained uncertain.
\end{abstract}

Methods: We conducted a meta-analysis of randomized controlled studies and prospective cohort studies to assess the effect of gargling with tea and its ingredients on the prevention of influenza infection. The published literature was searched using the Cochrane Library, PubMed/MEDLINE (1966 to September 2015), Web of Science (1981 to September 2015), and Ichu-shi Web (1983 to September 2015). The extracted studies were read by two reviewers independently, and their overall scientific quality was evaluated. Studies meeting our inclusion criteria were pooled using the Mantel-Haenszel method in a fixed effects model and were also analyzed in a random effects model. The qualities of the model fits were assessed using the Akaike information criterion (AIC) and Bayesian information criterion (BIC).

Results: The literature search and review identified 5 studies that met the inclusion criteria for the meta-analysis (total number of participants, 1890; mean age range, 16-83 years). The participants who gargled with tea or its ingredients showed a lower risk of influenza infection than did participants who gargled with placebo/water or who did not gargle (fixed effects model, Mantel-Haenszel method: relative risk $[R R]=0.70,95 \%$ confidence interval $[\mathrm{Cl}]=0.54-0.89$; random effects model: $R R=0.71,95 \% \mathrm{Cl}=0.56-0.91)$. The fixed effects model had a better quality of fit than the random effects model (fixed effects model: $\mathrm{AIC}=6.04, \mathrm{BIC}=5.65$; random effects model: $\mathrm{AIC}=8.74, \mathrm{BIC}=7.52$ ).

Conclusions: Gargling with tea and its ingredients may have a preventative effect for influenza infection. However, additional large-scale studies in different populations and a pooled analysis of these studies are needed to confirm the effect.

Keywords: Tea, Catechins, Influenza, Infection, Hygiene, Meta-analysis

\footnotetext{
*Correspondence: hyamada@u-shizuoka-ken.ac.jp

Department of Drug Evaluation \& Informatics, Graduate School of

Pharmaceutical Sciences, University of Shizuoka, 52-1 Yada, Suruga-ku,

Shizuoka 422-8526, Japan
} 


\section{Background}

Influenza viruses can spread easily from person to person, and annual epidemics create serious public health problems worldwide. The World Health Organization estimates that 5 to $10 \%$ of adults and 20 to $30 \%$ of children are infected by the virus annually, resulting in approximately 3 to 5 million cases of severe illness and 250,000 to 300,000 deaths [1]. Effective preventative measures are therefore needed to reduce serious cases of influenza.

Vaccination is a commonly used and widely recommended preventive measure for influenza infection $[1,2]$. Its effectiveness has been reported in both randomized controlled trials and meta-analyses [2, 3]. Nonetheless, there are several drawbacks to vaccinations for influenza viruses, including the limited supplies of vaccines [4-6] and their strain-specific effects [2, 3]. The effectiveness of influenza vaccination may also depend on the characteristics of the vaccinated population [7]. Neuraminidase inhibitors are also used for influenza prevention, but they have limited effects and the existence of resistant viruses has been reported $[8,9]$. Therefore, in addition to these pharmaceutical interventions, non-pharmaceutical public health interventions are also important for epidemic control, including measures such as the use of facemasks, hand hygiene, and gargling [10-12]. One meta-analysis study reported that a combination of hand hygiene with facemasks was effective for influenza prevention, even though hand hygiene was ineffective by itself [13]. Nonpharmaceutical public health interventions have the potential to be effective, but a combination of these interventions and/or improvements to their effectiveness would be needed to control influenza.

Tea catechins have been reported to have antiviral activities in in vitro and in vivo studies [14-16]. In vitro studies have suggested that (-)-epigallocatechin gallate, a highly bioactive catechin, reduces the infectivity of both influenza A and B viruses in Madin-Darby canine kidney cells $[14,15]$. Influenza $A$ and $B$ are mainly spread seasonally, and are particularly problematic to public health. Several clinical studies have also investigated the use of tea and its ingredients to prevent influenza infection [17-23]. These studies have examined both the effects of tea consumption and the use of tea as a gargle rinse. However, only a limited number of studies have focused on tea consumption [20,23], and the effect of gargling with tea or its ingredients has remained inconclusive. Indeed, although several small-scale studies have demonstrated efficacy $[17,18]$, other studies have not shown any significant effect $[19,21,22]$.

Considering the current state of research, we decided to conduct a meta-analysis of randomized controlled studies and prospective cohort studies to assess the effect of gargling with tea and its ingredients on the prevention of influenza infection.

\section{Methods}

\section{Design overview}

The primary endpoint of this study was influenza infection that had been confirmed by antigen detection testing and/or antibody titer testing. We searched the published literature using common electronic databases (Cochrane Library, PubMed/MEDLINE, Web of Science, and Ichu-shi Web), and additionally performed a manual search using the reference lists of the included studies. All studies were reviewed by two reviewers independently (KI and HY). The overall scientific quality of the randomized controlled studies was assessed using the Jadad score [24, 25] and the Cochrane Collaboration's tool for assessing risk for bias [26]. The non-randomized cohort studies were assessed using the NewcastleOttawa scale [27, 28]. The studies that were ultimately selected for our meta-analysis were pooled using the Mantel-Haenszel method [29] and a random effects model. Publication bias was assessed using a funnel plot [30] and Egger's regression analysis [31]. This study was a meta-analysis of previously published results and did not enroll any humans or animals. Accordingly, additional informed consent and ethical approval were not required.

\section{Search strategy and selection criteria}

The published literature was searched using the Cochrane Library (http://www.cochranelibrary.com/), PubMed/MEDLINE (1966 to September 2015), Web of Science (1981 to September 2015), and Ichu-shi Web (1983 to September 2015). This search was performed with the following terms in MEDLINE: ("catechin" [MeSH Terms] OR "tea” [MeSH Terms]) AND ("influenza, human" [MeSH Terms] OR "upper respiratory tract inflammation" [All Fields]) AND gargling [All Fields] AND (clinical trial [pt] OR "clinical trials as topic" [MeSH Terms: noexp] OR trial [ti]) AND Clinical Trial [ptyp]. In addition, the reference lists of the included studies were searched manually.

All studies extracted from the databases were independently reviewed by two reviewers (KI and HY). As noted above, the randomized controlled studies were assessed using the Jadad score $[24,25]$ and the Cochrane Collaboration's tool for assessing risk for bias [26]. The Jadad score is calculated by assessing randomization (range, 0-2), double blinding (range, 0-2), and withdrawals and dropouts (range, 0-1). The total score ranges from 0 to 5 and is interpreted according to the following criteria: 0-2 indicates a low-quality report and 3-5 indicates a high-quality report. The nonrandomized cohort studies were assessed using the Newcastle-Ottawa scale $[27,28]$. This scale is constructed from 3 grouping items, and stars are awarded for each item: 1) selection of cohorts (range, 0-4 stars), 2) comparability of cohorts (range, 0-2 stars), and 3) assessment of outcomes (range, 0-3 stars). The total number of stars 
ranges from 0 to 9 , and a high quality study can be awarded 9 stars.

The meta-analysis included studies that met each of the following criteria: 1) studies with randomized controlled designs or prospective cohort designs; 2) studies that investigated gargling with tea or its components for at least 60 days; 3) studies with a control group, such as a placebo, water, or non-gargling group; 4) studies in which influenza infection was confirmed by antigen and/ or antibody detection methods; 5) studies published in English and/or Japanese. The criterion regarding the duration of the gargling was chosen based on seasonal epidemic periods.

\section{Data extraction}

The following information was extracted from published reports: 1) the authors' names and the year of publication, 2) the study population, 3) the study design and the duration of the intervention or observation, and 4) the number of participants infected by influenza and the methods of confirmation.

\section{Endpoint}

The primary endpoint of this study was influenza infection that had been confirmed by an antigen detection test. In addition to rapid antigen tests that are used in clinical practice, we also included changes in antibody titer in this endpoint.

\section{Statistical analyses}

The studies that were selected for the meta-analysis were pooled using both the Mantel-Haenszel method for a fixed effects model and a random effects model $[32,33]$. The random effects model was fitted via restricted maximum-likelihood estimation. The results were expressed as relative risks (RRs) with $95 \%$ confidence intervals (CIs). Heterogeneity among the studies was assessed using a $Q$-test and quantified with the $I^{2}$ statistic for the random effects model [34]. For the $Q$-test, $P<0.1$ was considered representative of statistically significant heterogeneity. $I^{2}$ estimates the total percentage of variability in the effect size estimates, with $I^{2}>50 \%$ indicating moderate to large heterogeneity. The relative qualities of model fits were assessed using the Akaike information criterion (AIC) [35] and the Bayesian information criterion (BIC) [36]. AIC and $\mathrm{BIC}$ values were compared between the fixed and random effects models to judge their relative qualities, with smaller values of AIC and BIC indicating better fits of the model to the data $[35,36]$. Publication bias was assessed using a funnel plot [30] and Egger's regression analysis [31]. For the Egger's regression analysis, $P<0.1$ was considered representative of statistically significant publication bias. All statistical analyses were performed using $\mathrm{R}$ version 3.2.0 for Windows with the Metafor package [37] (The R Foundation for Statistical Computing, Vienna, Austria).

\section{Results}

Description of the included studies

The flow diagram for the meta-analysis is shown in Fig. 1. Seventeen studies were identified in the literature search ( 8 from the Cochrane Library, 3 from PubMed/ Medline, 2 from Web of Science, and 4 from Ichu-shi Web). Twelve studies were excluded because they were duplicated or did not present original data on the efficacy of gargling. The 5 remaining studies were included in the meta-analysis. Of the included studies, 2 had prospective cohort designs and 3 were randomized controlled trials. The characteristics of the studies, such as the publishing details (author and year), study populations, and study designs, are shown in Table 1. The total

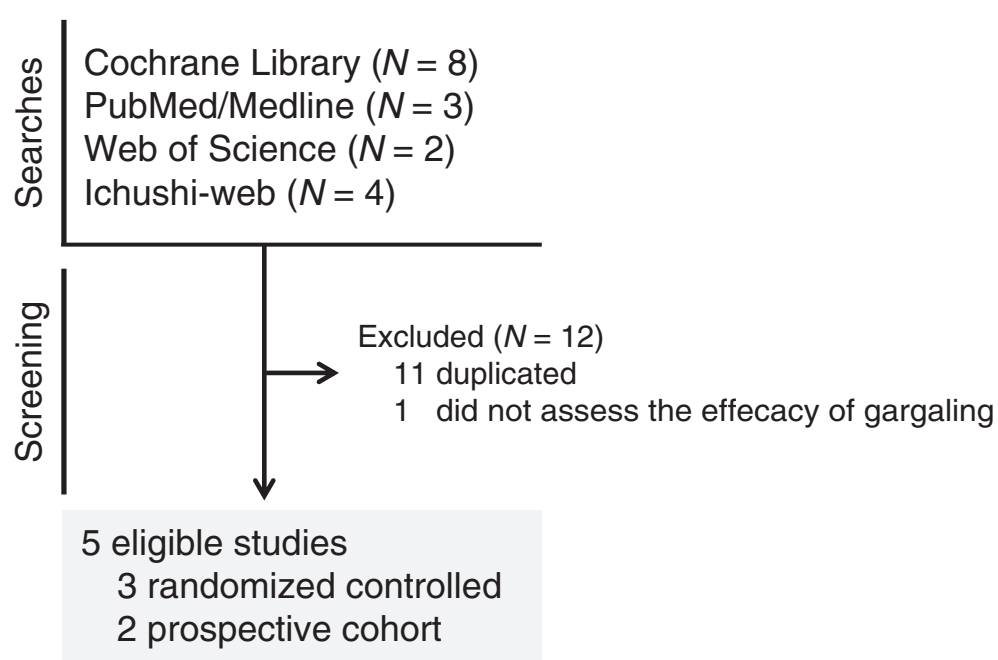

Fig. 1 Flow diagram for the study 
Table 1 Characteristics of the included studies

\begin{tabular}{|c|c|c|c|c|c|c|c|c|c|}
\hline \multirow[b]{2}{*}{ Author } & \multirow[b]{2}{*}{ Year of publication } & \multirow[b]{2}{*}{ Total $N$} & \multicolumn{2}{|c|}{ Age $(y r)$, mean $\pm S D$} & \multicolumn{2}{|l|}{$\operatorname{Sex}(M / F)$} & \multirow[t]{2}{*}{ Duration (mo) } & \multirow[t]{2}{*}{ Study design } & \multirow[t]{2}{*}{ Reference } \\
\hline & & & Tea & Control & Tea & Control & & & \\
\hline Iwata et al. & 1997 & 297 & $30 \pm N A$ & $24 \pm N A$ & NA & NA & 5 & Prospective cohort & [17] \\
\hline Yamada et al. & 2006 & 124 & $83 \pm 8$ & $83 \pm 8$ & $24 / 52$ & $16 / 32$ & 3 & Prospective cohort & [18] \\
\hline Yamada et al. & 2007 & 404 & $40 \pm 11$ & $40 \pm 12$ & $36 / 164$ & $52 / 152$ & 3 & Randomized controlled & [19] \\
\hline Toyoizumi et al. & 2013 & 308 & $16 \pm 1$ & $16 \pm 1$ & $98 / 57$ & $86 / 67$ & 3 & Randomized controlled & [21] \\
\hline Ide et al. & 2014 & 757 & $16 \pm 1^{*}$ & $16 \pm 1^{*}$ & $224 / 160^{*}$ & $199 / 164^{*}$ & 3 & Randomized controlled & {$[22]$} \\
\hline
\end{tabular}

yr years, $S D$ standard deviation, $M$ male, $F$ female, mo months, $N A$ not assessed.

* Full analysis set (Characteristics of the total population is not shown in the article)

number of participants in each study ranged from 124 to 757 , and the range of mean ages was 16 to 83 years. The durations of the studies ranged from 3 to 5 months. In the randomized controlled studies, the active groups received either green tea extract (catechin solution, 1 study) or bottled green tea ( 2 studies), and the control groups received either placebo (1 study) or water (2 studies). One cohort study selected the exposed cohort from the population of persons with a habit of gargling green tea. The other cohort study selected the exposed cohort from the population of persons with a habit of gargling black tea. The 2 cohort studies selected the non-exposed cohort from the same population-persons with or without a habit of gargling water. All of the 5 studies were conducted in Japan.

Regarding the overall scientific quality of the studies, the 2 prospective cohort studies were awarded 7 stars on the Newcastle-Ottawa scale, and the 3 randomized controlled studies received Jadad scores of 3 to 5 . The results of the assessments using the Newcastle-Ottawa scale are shown in Table 2 and, the results of those using the Cochrane Collaboration's tool for assessing risk for bias and the Jadad score are shown in Table 3.

\section{Heterogeneity}

The Q-test and $I^{2}$ statistic both suggested low heterogeneity among the studies included in this meta-analysis $\left(I^{2}=0.00 \%\right.$; $Q$-test: $\left.Q=3.05, P=0.55\right)$.

\section{Efficacy assessment}

A forest plot of the RRs for influenza infection is shown in Fig. 2a, b. The 5 studies included a total of 1890

Table 2 Overall scientific quality of the prospective cohort studies

\begin{tabular}{lll}
\hline Newcastle-Ottawa scale & & \\
\hline Study & Iwata et al. 1997 & Yamada et al. 2006 \\
Criterion & {$[17]$} & {$[18]$} \\
\hline Selection (range, 0-4 stars) & $* * *$ & $* * *$ \\
Comparability (range, 0-2 stars) & $*$ & $*$ \\
Outcome (range, 0-3 stars) & $* * *$ & $* * *$ \\
\hline
\end{tabular}

* One asterisk represents one star participants. The participants who gargled with tea or its ingredients showed a lower risk of influenza infection than did the participants who gargled with placebo/ water or who did not gargle (fixed effects model: $R R=$ $0.70,95 \% \mathrm{CI}=0.54-0.89$; random effects model: $\mathrm{RR}=$ $0.71,95 \% \mathrm{CI}=0.56-0.91)$. The results were consistent between the fixed effects model (Fig. 2a) and the random effects model (Fig. 2b). The qualities of the model fits were as follows: the fixed effects model had an AIC of 6.04 and a BIC of 5.65, while the random effects model had an AIC of 8.74 and a BIC of 7.52. Accordingly, the fixed effects model showed a better quality of fit to the data, as compared with the random effects model.

\section{Potential publication bias}

A funnel plot of the included studies is shown in Fig. 3. Because of the limited number of studies that were included in this analysis $(n=5)$, visual interpretation of the plot was inconclusive. The result of the Egger's regression analysis was not significant $(P=0.30)$.

Table 3 Overall scientific quality of the randomized controlled studies

\begin{tabular}{|c|c|c|c|}
\hline Study & $\begin{array}{l}\text { Yamada } \\
\text { et al. } 2007\end{array}$ & $\begin{array}{l}\text { Toyoizumi } \\
\text { et al. } 2013\end{array}$ & $\begin{array}{l}\text { Ide } \\
\text { et al. } 2014\end{array}$ \\
\hline Criterion & [19] & [21] & [22] \\
\hline \multicolumn{4}{|c|}{ Cochrane Collaboration's tool for assessing risk for bias } \\
\hline Random sequence generation & Low & Low & Low \\
\hline Allocation concealment & Low & High & High \\
\hline Blind participants and personnel & Low & High & High \\
\hline Blind outcome assessment & Low & Low & Low \\
\hline Incomplete outcome data & Low & Low & Low \\
\hline Selective reporting & Low & High & High \\
\hline Other bias & Low & Unclear & Unclear \\
\hline \multicolumn{4}{|l|}{ Jadad score } \\
\hline Randomization (range, 0-2) & 2 & 2 & 2 \\
\hline Double blinding (range, 0-2) & 2 & 0 & 0 \\
\hline $\begin{array}{l}\text { Withdrawals and dropouts } \\
\text { (range, } 0-1 \text { ) }\end{array}$ & 1 & 1 & 1 \\
\hline Total score & 5 & 3 & 3 \\
\hline
\end{tabular}




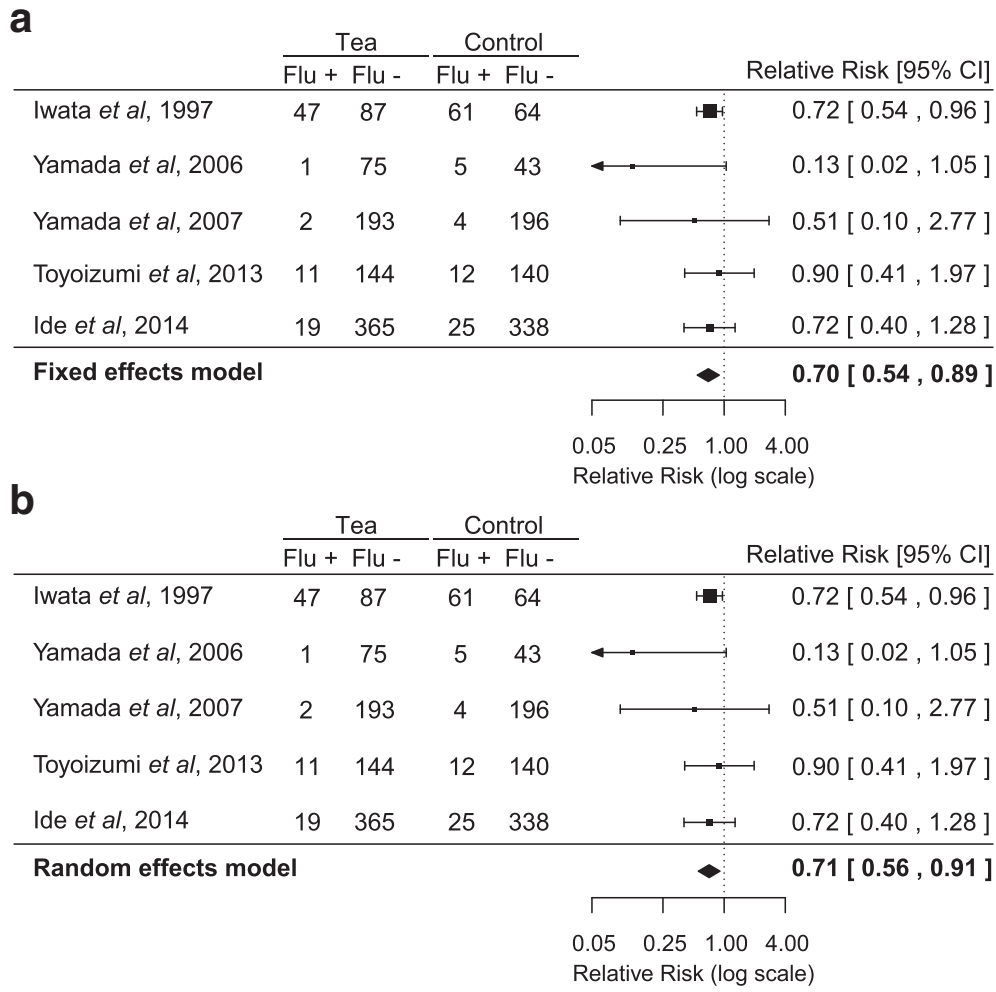

Fig. 2 Forest plot of fixed effects model (a) and random effects model (b)

\section{Discussion}

The meta-analysis of 5 studies with 1890 participants indicated that gargling with tea and its ingredients reduces the risk of influenza infection, as compared with the control activities. The effects of gargling with tea and its ingredients on the prevention of influenza infection have been investigated since the 1990s; nonetheless, the present study is the first meta-analysis to consider this subject. A combination of non-pharmaceutical interventions and/or an improvement in the effectiveness of non-pharmaceutical interventions are needed to control influenza epidemics [13]. Tea and its ingredients may be useful for this purpose. The mean ages of participants in the included studies ranged from 16 to 83 years, and this suggested that gargling with tea and its ingredients might be effective for any age group or population. All of the studies included in the analysis used a control group or non-exposed cohort from the same population, which ensures the comparability of their results. In addition, all of the cohort studies and randomized controlled trials were well designed [17-19, 21, 22]. Therefore, the results of this meta-analysis of well-designed studies may be reliable. The AIC and BIC values for the fixed effects model were smaller than those for the random effects model, which indicates that the fixed effects model had a better fit.
Although the meta-analytic results of this study suggest that gargling with tea and its ingredients has efficacy for influenza prevention, there are several limitations to our study. A principal limitation is the small number of studies that met the inclusion criteria. Further, all 5 of these studies were conducted in Japan, which could bias or limit interpretations of the findings. The fact that all of the studies were conducted in Japan may have helped to avoid between-study heterogeneity, which is often caused by the inclusion of different populations with different sociodemographic characteristics. Nonetheless, additional studies of different populations would still be needed to improve the generalizability of the meta-analytic results. If the number of studies were increased, a sensitivity analysis could also be performed. Sensitivity analyses can provide indications of the robustness of meta-analytic results, including insight into factors that affect the efficacy of gargling with tea and its ingredients [38, 39]. A sensitivity analysis should be carried out in future pooled analyses with additional high-quality studies. As additional highquality studies become available in the future, it may also be possible to consider more sophisticated methods for the meta-analysis. We used standard methods for the meta-analysis in the present study $[32,33]$. However, several newer approaches are also available, such as individual participant data meta-analysis [Riley 2010] and Bayesian 


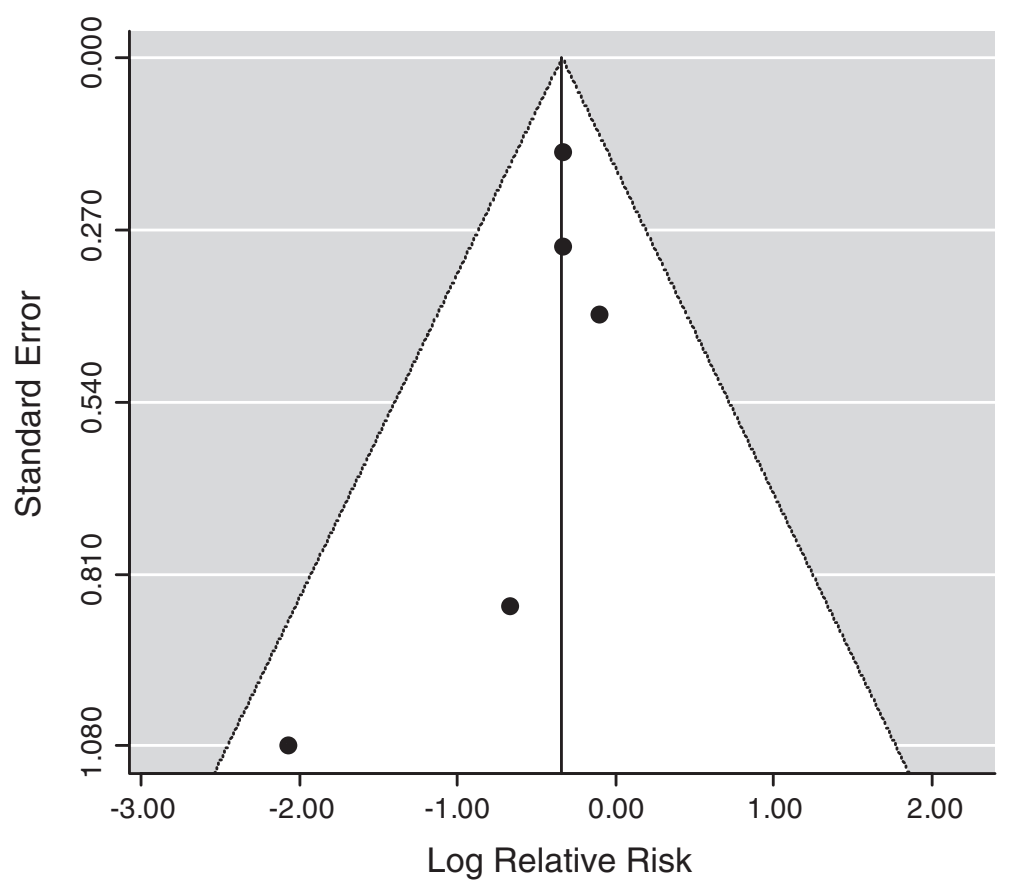

Fig. 3 Funnel plot

meta-analysis [Sutton 2008]. If future meta-analytic studies apply both standard and new approaches, then they could offer more reliable results and greater information about the robustness of the meta-analytic conclusions. The quality of the present study could also be improved by expanding the analysis to additional databases, and by collecting data from other Asian countries. Although we searched 5 major databases for the present study, there are several databases that were not searched, such as EMBASE.

With respect to publication bias, the result of the Egger's regression analysis was not significant and therefore did not suggest significant publication bias. Nonetheless, visual interpretation of the funnel plot was inconclusive. The results may have been affected by the small number of studies that were pooled in the analysis, and additional studies are needed to obtain more definitive conclusions regarding publication bias. In the future, additional large-scale randomized controlled studies and a pooled analysis of these studies could help to improve the accuracy and robustness of the effect estimate for gargling with tea and its ingredients.

\section{Conclusions}

Gargling with tea or ingredients of tea may be effective for the prevention of influenza infection. Given the effectiveness that has been shown in this meta-analysis, gargling with tea or its ingredients may provide a simple and useful addition to the non-pharmaceutical interventions that are currently employed for influenza control. However, additional large-scale randomized controlled trials of different populations are needed to confirm our findings.

\section{Ethics and consent to participate}

This study was a meta-analysis of previously published results and did not enroll any humans or animals. Accordingly, additional informed consent and ethical approval were not required.

\section{Consent for publication}

Not Applicable.

\section{Availability of data and materials}

No additional data available.

\section{Abbreviations}

AIC: Akaike information criterion; BIC: Bayesian information criterion; $\mathrm{Cl}$ : confidence interval; $\mathrm{RR}$ : relative risk.

\section{Competing interests}

The authors declare that they have no competing interests.

\section{Authors' contributions}

$\mathrm{KI}$ and $\mathrm{YK}$ had full access to all of the data in the study and take responsibility for the integrity of the data and the accuracy of the data analysis. Study concept and design: Kl. Acquisition of data: KI, HY. Statistical analysis and interpretation of data: Kl, YK. Writing of the manuscript: Kl. All authors reviewed and approved the contents of the manuscript.

\section{Acknowledgement}

This work was supported in part by a grant from the Japan Society for the Promotion of Science (JSPS), through the Grant-in-Aid for JSPS Fellows (No. $15 \mathrm{~J} 10190$ to $\mathrm{KI})$. 
Received: 14 October 2015 Accepted: 6 May 2016

\section{Published online: 12 May 2016}

\section{References}

1. WHO. Influenza (Seasonal). Fact Sheet No 211 2014. http://www.who.int/ mediacentre/factsheets/fs211/en/. Accessed 13 Oct 2015.

2. Osterholm MT, Kelley NS, Sommer A, Belongia EA. Efficacy and effectiveness of influenza vaccines: a systematic review and meta-analysis. Lancet Infect Dis. 2012;12:36-44.

3. DiazGranados CA, Denis M, Plotkin S. Seasonal influenza vaccine efficacy and its determinants in children and non-elderly adults: a systematic review with meta-analyses of controlled trials. Vaccine. 2012;31:49-57.

4. Partridge J, Kieny MP. Global production capacity of seasonal influenza vaccine in 2011. Vaccine. 2013;31:728-31.

5. Collin N, de Radigues X. World Health Organization H1N1 Vaccine Task Force. Vaccine production capacity for seasonal and pandemic (H1N1) 2009 influenza. Vaccine. 2009;27:5184-6.

6. Duque J, McMorrow ML, Cohen AL. Influenza vaccines and influenza antiviral drugs in Africa: are they available and do guidelines for their use exist? BMC Public Health. 2014;14:41.

7. Ohmit SE, Petrie JG, Malosh RE, Cowling BJ, Thompson MG, Shay DK, et al. Influenza vaccine effectiveness in the community and the household. Clin Infect Dis. 2013;56:1363-9.

8. Jefferson T, Jones M, Doshi P, Del Mar C. Neuraminidase inhibitors for preventing and treating influenza in healthy adults: systematic review and meta-analysis. BMJ. 2009:339:b5106.

9. Jefferson T, Jones MA, Doshi P, Del Mar CB, Hama R, Thompson MJ, et al. Neuraminidase inhibitors for preventing and treating influenza in healthy adults and children. Cochrane Database Syst Rev. 2014;4:CD008965.

10. Aiello AE, Murray GF, Perez V, Coulborn RM, Davis BM, Uddin M, et al. Mask Use, Hand Hygiene, and Seasonal Influenza-Like Illness among Young Adults: A Randomized Intervention Trial. J Infect Dis. 2010;201:491-8.

11. Aiello AE, Perez V, Coulborn RM, Davis BM, Uddin M, Monto AS. Facemasks, hand hygiene, and influenza among young adults: a randomized intervention trial. PLoS One. 2012:7:e29744.

12. Godoy P, Castilla J, Delgado-Rodriguez M, Martin V, Soldevila N, Alonso J, et al. Effectiveness of hand hygiene and provision of information in preventing influenza cases requiring hospitalization. Prev Med. 2012;54:434-9.

13. Wong WW, Cowling BJ, Aiello AE. Hand hygiene and risk of influenza virus infections in the community: a systematic review and meta-analysis. Epidemiol Infect. 2014;142:922-32.

14. Steinmann J, Buer J, Pietschmann T, Steinmann E. Anti-infective properties of epigallocatechin-3-gallate (EGCG), a component of green tea. $\mathrm{Br}$ Pharmacol. 2013;168:1059-73.

15. Song JM, Seong BL. Anti-Influenza Viral Activity of Catechins and Derivatives. In: Preedy VR, editor. Tea in Health and Disease Prevention. San Diego: Academic; 2013. p. 1185-93.

16. Lee HJ, Lee $\mathrm{YN}$, Youn HN, Lee DH, Kwak JH, Seong BL, et al. Anti-influenza virus activity of green tea by-products in vitro and efficacy against influenza virus infection in chickens. Poult Sci. 2012:91:66-73.

17. Iwata M, Toda M, Nakayama M, Tsujiyama H, Endo W, Takahashi O, et al. Prophylactic effect of black tea extract as gargle against influenza. Kansenshogaku zasshi. 1997;71:487-94.

18. Yamada H, Takuma N, Daimon T, Hara Y. Gargling with tea catechin extracts for the prevention of influenza infection in elderly nursing home residents: a prospective clinical study. J Altern Complement Med. 2006;12:669-72.

19. Yamada H, Daimon T, Matsuda K, Yoshida M, Takuma N, Hara Y. A randomized controlled study on the effects of gargling with tea catechin extracts on the prevention of influenza infection in healthy adults. Jpn J Clin Pharmacol Ther. 2007;38:323-30.

20. Matsumoto K, Yamada H, Takuma N, Niino H, Sagesaka YM. Effects of green tea catechins and theanine on preventing influenza infection among healthcare workers: a randomized controlled trial. BMC Complement Altern Med. 2011;11:15

21. Toyoizumi K, Yamada H, Matsumoto K, Sameshima Y. Gargling with Green Tea for Influenza Prophylaxis: A Pilot Clinical Study. Jpn J Clin Pharmacol Ther. 2013:44:459-61.

22. Ide K, Yamada H, Matsushita K, Ito M, Nojiri K, Toyoizumi K, et al. Effects of green tea gargling on the prevention of influenza infection in high schoo students: A randomized controlled study. PLoS One. 2014;9:e96373.
23. Park M, Yamada H, Matsushita K, Kaji S, Goto T, Okada Y, et al. Green Tea Consumption Is Inversely Associated with the Incidence of Influenza Infection among Schoolchildren in a Tea Plantation Area of Japan. J Nutr. 2011;141:1862-70.

24. Jadad AR, Moore RA, Carroll D, Jenkinson C, Reynolds DJ, Gavaghan DJ, et al. Assessing the quality of reports of randomized clinical trials: Is blinding necessary? Control Clin Trials. 1996;17:1-12.

25. Moher D, Jadad AR, Nichol G, Penman M, Tugwell P, Walsh S. Assessing the quality of randomized controlled trials: an annotated bibliography of scales and checklists. Control Clin Trials. 1995;16:62-73.

26. Higgins JP, Altman DG, Gotzsche PC, Juni P, Moher D, Oxman AD, et al. The Cochrane Collaboration's tool for assessing risk of bias in randomised trials. BMJ. 2011:343:d5928.

27. Stang A. Critical evaluation of the Newcastle-Ottawa scale for the assessment of the quality of nonrandomized studies in meta-analyses. Eur J Epidemiol. 2010;25:603-5.

28. Zeng X, Zhang Y, Kwong JS, Zhang C, Li S, Sun F, et al. The methodological quality assessment tools for preclinical and clinical studies, systematic review and meta-analysis, and clinical practice guideline: a systematic review. J Evid Based Med. 2015:8:2-10.

29. Mantel N, Haenszel W. Statistical aspects of the analysis of data from retrospective studies of disease. J Natl Cancer Inst. 1959:22:719-48.

30. Duval S, Tweedie R. Trim and fill: A simple funnel-plot-based method of testing and adjusting for publication bias in meta-analysis. Biometrics. 2000; 56:455-63.

31. Egger M, Davey Smith G, Schneider M, Minder C. Bias in meta-analysis detected by a simple, graphical test. BMJ. 1997;315:629-34.

32. Harville DA. Maximum likelihood approaches to variance component estimation and to related problems. J Am Stat Assoc. 1977;72:320-38.

33. DerSimonian $\mathrm{R}$, Kacker $\mathrm{R}$. Random-effects model for meta-analysis of clinical trials: an update. Contemp Clin Trials. 2007:28:105-14.

34. Higgins JP, Thompson SG. Quantifying heterogeneity in a meta-analysis. Stat Med. 2002;21:1539-58.

35. Akaike H. A new look at the statistical-model identification. IEEE Trans Automat Contr. 1974:19:716-23.

36. Schwarz G. Estimating the dimension of a model. Ann Stat. 1978;6:461-4.

37. Viechtbauer W. Conducting Meta-Analyses in $\mathrm{R}$ with the metafor Package. J Stat Softwear. 2010;36:1-48.

38. Crowther M, Lim W, Crowther MA. Systematic review and meta-analysis methodology. Blood. 2010;116:3140-6.

39. Donner A, Klar N. Issues in the meta-analysis of cluster randomized trials. Stat Med. 2002:21:2971-80.

\section{Submit your next manuscript to BioMed Central and we will help you at every step:}

- We accept pre-submission inquiries

- Our selector tool helps you to find the most relevant journal

- We provide round the clock customer support

- Convenient online submission

- Thorough peer review

- Inclusion in PubMed and all major indexing services

- Maximum visibility for your research

Submit your manuscript at www.biomedcentral.com/submit
) Biomed Central 